\title{
A case of multiple organ dysfunction syndrome after large area burns resulting from sustained relatively low heat
}

\author{
Shengjun Cao, Lingfeng Wang, Te Ba \\ The Third Affiliated Hospital of Inner Mongolia Medical University, Baotou, China
}

Received: October 20, 2016

DOI: $10.14725 /$ dcc.v3n4p18
Accepted: November 21, $2016 \quad$ Online Published: December 10, 2016

URL: http://dx.doi.org/10.14725/dcc.v3n4p18

\begin{abstract}
Burn as a strong traumatic stimulus can induce inflammatory responses mediated by neuroendocrine, cytokines and other inflammatory mediators, and directly lead to organ dysfunction, resulting in severe MODS. Excessive inflammatory responses caused by necrotic tissues in the burned wound, also play an important role in stimulating the occurrence and the development of MODS. Complete removal of necrotic tissues in the early stage of burns to prevent from invasive wound infections, can effectively prevent MODS. AKI occurred earlier in this case, combined with its admission after the onset of shock and no obstruction of the urinary system can exclude prerenal and renal AKI, speculated as renal AKI. The factors leading to renal AKI are divided into renal ischemia and nephrotoxicity. The patient had adequate urinary output during shock and AKI in the presence of adequate renal blood flow, possibly due to the nephrotoxicity of a large number of necrotic tissues of the wound after they were broken down and absorbed into the bloodstream. The patient fell asleep after being drunk and the bedside stove broiled him for a long time, on one hand, heat accumulation lead to skin damage; on the other hand, sustained heat action caused hyperemia, edema and hemodynamic disorder in the deep tissues, and then resulted in secondary necrosis.
\end{abstract}

Key Words: MODS, AKI, necrosis

\section{Case presentation}

The patient, male, 50 years old, was asleep after being drunk On January 17, 2007. He warmed himself by the bedside stove continuously for several hours. After the fire, he was rescued and admitted to hospital 4 hours later. On admission, the patient was of clear consciousness with eschar wounds (red and white basement) scattered on the trunk and limbs. The results of physical examination: body temperature $36.7^{\circ} \mathrm{C}$, pulse 132 times/min, respiratory rate 24 times $/ \mathrm{min}$, blood pressure $135 / 90 \mathrm{mmHg}(1 \mathrm{mmHg}=0.133$ $\mathrm{kPa})$. Laboratory tests: serum sodium $149.6 \mathrm{mmol} / \mathrm{L}$, creatinine $44.5 \mu \mathrm{mol} / \mathrm{L}$, blood urea nitrogen $4.4 \mathrm{mmol} / \mathrm{L}$. Admission diagnosis: flame burns 50\% TBSA: $25 \%$ III degree burns, 25\% deep TBSA.
After admission, the patient was given fluid therapy for antishock and given cefoperazone/sulbactam ( $3 \mathrm{~g}$ per time, 3 times per day) by ingtt for 7 days for the purpose of antiinfection. The exposed wounds were treated with povidoneiodine. In the first 24 hours after injury, $4,430 \mathrm{~mL}$ of electrolyte and 3,500 $\mathrm{mL}$ of colloid were transfused, and 1,290 $\mathrm{mL}$ of urine was collected. The results of laboratory tests were as follows: serum sodium $139.4 \mathrm{mmol} / \mathrm{L}$, creatinine $285.5 \mu \mathrm{mol} / \mathrm{L}$, blood urea nitrogen $12.2 \mathrm{mmol} / \mathrm{L}$. According to classification criteria of acute kidney injury (AKI), ${ }^{[1]}$ the supplementary diagnosis was Phase II (Impair phase). The transfusion volume of colloid (plasma) was increased, and furosemide was given by intravenous injection for diuresis for three days (given every 8 hours with $20 \mathrm{mg}$ per time).

\footnotetext{
*Correspondence: Lingfeng Wang; E-mail: wlf7413@vip.sina.com; Address: The Third Affiliated Hospital of Inner Mongolia Medical University, Baotou, China.
} 
The exposed wounds were treated with silver sulfadiazine. In the second 24 hours after injury, $2,800 \mathrm{~mL}$ of electrolyte and 2,800 $\mathrm{mL}$ of colloid were transfused, and 1,790 $\mathrm{mL}$ of urine was collected. The results of laboratory tests were as follows: serum sodium $144.3 \mathrm{mmol} / \mathrm{L}$, creatinine 332.4 $\mu \mathrm{mol} / \mathrm{L}$, blood urea nitrogen $20.1 \mathrm{mmol} / \mathrm{L}$.

On the third day after injury, 33\% TBSA wounds on the limbs were given treatment with tangential excision. Scalp graft, micro-skin graft and allogenic skin graft (donated by volunteers) were also conducted on the limbs. In the operation of tangential excision, intact dermis and subcutaneous tissues observed by the naked eyes were preserved. On the next day after operation, the patient was of clear consciousness, with the temperature $37.5^{\circ} \mathrm{C}$, respiratory rate 24 beats/min, pulse 105 beats/min, blood pressure $168 / 95 \mathrm{mmHg}$. The results of laboratory tests were as follows: serum sodium $159.6 \mathrm{mmol} / \mathrm{L}$, creatinine 475.2 $\mu \mathrm{mol} / \mathrm{L}$, blood urea nitrogen $41.2 \mathrm{mmol} / \mathrm{L}$, AKI was exacerbated to Phase III (exhaustion phase). ${ }^{[1]}$ During the treatment, the intake of salt and liquid was strictly controlled, and furosemide was given by intravenous injection for 2 days (given every 2 hours with $40 \mathrm{mg}$ per time). Meanwhile, Prisma continuous blood purification machine (Gambro Dasco S.P.A, Italy) was applied to the bedside continuous renal replacement therapy ( 8 hours per time, one time every day, for 2 days). The operation mode was continuous vena-venous hemofiltration (CVVH). Signo catheter was indwelt in the femoral vein on one side, and $312.5 \mathrm{U} / \mathrm{mL}$ of LMWH SODIUM $(1 \mathrm{~mL} / \mathrm{h})$ was used for anticoagulation.

On the $4^{\text {th }}$ day after surgery, the patient fell in a restless state or in a sopor state sometimes. The patient coughed with a large amount of pink frothy sputa, and also showed other symptoms of bloody stools, continuous fever (above $39.0^{\circ} \mathrm{C}$ ), pulse $135-150$ times/min, respiratory rate 35 to 42 times/min, blood pressure $170 / 100 \mathrm{mmHg}$, central venous pressure $12-18 \mathrm{cmH}_{2} \mathrm{O}\left(1 \mathrm{cmH}_{2} \mathrm{O}=0.098 \mathrm{kPa}\right)$, moist crackles on double lungs, $\mathrm{SaO}_{2}$ 0.8-0.9. $\mathrm{PaO}_{2} 51.4 \mathrm{mmHg}$, $\mathrm{PaCO}_{2} 21.8 \mathrm{mmHg}$, creatinine $566.0 \mu \mathrm{mol} / \mathrm{L}$, blood urea nitrogen $69.8 \mathrm{mmol} / \mathrm{L}, \mathrm{FOBT}+++$. The patient developed ARDS, congestive heart failure and alimentary tract hemorrhage, in consistent with the diagnosis of MODS. ${ }^{[2]}$ From the $4^{t h}$ day after operation, on the basis of the original treatment, the frequency of continuous renal replacement therapy was changed to 2 times/d (the method was same as the above), and the treatment was continued for 5 days. Besides, the daily dehydration was calculated according to intake and output volume as well as central venous pressure. The patient's trachea was incised with the application of PB840 ventilator (Tyco, USA). The mode was synchronized intermittent mandatory ventilation (SIMV), and the parameters were adjusted according to the levels of $\mathrm{SaO}_{2}$ and $\mathrm{PaO}_{2}$. Imipenem/cilastatin (for injection) was injected every 8 hours $(1 \mathrm{~g})$ and norvancomycin hydrochloride (for injection) was injected every 12 hours $(0.4 \mathrm{~g})$ for 6-day continuous anti-infective treatment. Injectable omeprazole was pumped continuously $(5 \mathrm{mg} / \mathrm{h})$ to inhibit gastric acid secretion.

On the $9^{\text {th }}$ day after the first operation, the patient showed no improvement in symptoms in all systems. Considering that the debridement may not be conducted thoroughly at the first time, the debridement was performed again on $33 \%$ TBSA wounds on the limbs, and the medium thickness skin was grafted from the back trunk and made into reticular skin. Reticular skin graft and pig ADM (acellular dermal matrix) were performed on the limbs. In the surgery, it was observed that liquefaction and necrosis occurred in a large amount of subcutaneous tissues under the allogenic skin, with no survival of micro-skin. The liquefactive and necrotic subcutaneous tissues were removed to the layer of deep fascia. On the next day after operation, the patient showed an improvement in symptoms with the urine volume gradually increased, the indicators of renal function returning to normal, moist crackles gradually disappearing, the levels of $\mathrm{SaO}_{2}$ and $\mathrm{PaO}_{2}$ recovering to normal, no more bloody stools and the condition keeping stable. Therefore, the treatment was gradually simplified. The residual wounds were treated by surgery again with the closure of autologous scalp graft. The patient was discharged from hospital in 2 months after injury.

\section{Discussion}

Burns, as a type of strong traumatic stimulation, can induce inflammatory responses mediated by neuroendocrine, cytokines and other inflammatory mediators, and directly lead to organ dysfunction, ${ }^{[3]}$ even MODS. Excessive inflammatory responses caused by necrotic tissues in the burned wound, also play an important role in stimulating the occurrence and the development of MODS. ${ }^{[4]}$ Complete removal of necrotic tissues in the early stage of burns to prevent from invasive wound infections, can effectively prevent MODS. ${ }^{[5,6]}$ AKI occurred earlier in this case. Considering that the patient showed no symptoms of shock and obstruction in the urinary system after admission, it was speculated as renal AKI with the exclusion of pre-renal AKI and postrenal AKI. Factors that cause renal AKI can be divided into 2 types: renal ischemia and renal toxicity. ${ }^{[7]}$ The patient developed AKI in the shock stage under the condition of fair urine volume and sufficient renal blood, it was probably because a large amount of necrotic wound tissues were decomposed and then absorbed by the renal blood, which consequently resulted in renal toxicity. The patient fell asleep after being drunk and the bedside stove broiled him for a long time, on one hand, heat accumulation lead to skin damage; on the other hand, sustained heat action caused hyperemia, edema and hemodynamic disorder in the deep tissues, and then resulted in secondary necrosis. ${ }^{[8]}$ In the first surgery of tangential excision, although the necrotic crustae were removed, parabiosis tissues in the stasis band of deep burns 
were not thoroughly eliminated. Metabolites generated by the decomposition of secondary necrosis had a continuous damage to the renal function after operation and caused excessive inflammatory responses, which activated inflammatory cells and endothelial cells so that cells adhered to and aggregated in the capillaries of organ tissues, impeded capillary blood flow, exacerbated micro-circulatory disturbance and tissue ischemia \& hypoxia. ${ }^{[9]}$ Consequently, it will have a second strike to the patient with multiple organ dysfunction gradually. The second surgery was performed under the condition of ineffective treatment and gradual exacerbation of symptoms. In the surgery, it was observed that necrosis and liquefaction occurred in a large amount of subcutaneous tissues under the allogenic skin. The liquefactive and necrotic subcutaneous tissues were removed and the wounds were closed with reticular skin by graft during the surgery. The patient showed an improvement in symptoms immediately after operation.

Sustained heat action could have a damage to the deep tis- sues, which formed a stasis band. Parabiosis tissues in the stasis band cannot be seen exactly by the naked eyes in the surgery, which may easily lead to incomplete debridement. The tissues in the deep burned wounds caused by this relatively low sustained heat should be resected rather than excised. The reason for the exacerbation in this case, was that the physician failed to fully consider the main cause of injury to perform a debridement thoroughly. It is suggested that clinical physicians should pay more attention to the patients with the history of toxication, ebriety and coma, as well as inquire the medical history of patients carefully to determine if the relatively low sustained heat action exists, so that it is possible to perform a debridement thoroughly in the operation and avoid the secondary necrosis in parabiosis tissues of the stasis band and MODS.

\section{Conflicts of Interest Disclosure}

The authors have no conflicts of interest related to this article.

[5] Tu JJ, Fang RH, Zhou H, et al. Improvement effects of shaving or excising eschar in deeply burned patients on the renal function. Chinese Journal of Burns. 2006; 22(5): 373.

[6] Yao YM, Liu F, Sheng ZY. Strategies on multiple organ dysfunction syndrome and organ function support. Chinese Journal of Emergency Medicine. 2006; 15(4): 293-294.

[7] Duan SB, Zhang H, Peng YM. Pathogenesis and prevention of acute kidney injury. Chinese Journal of Blood Purification. 2010; 9(7): 349-351.

[8] Wang WY, Niu XH, Tian SM, et al. Experimental study on the mechanism of deep burns caused by sustained action of "relatively low heat". Henan Medical Research. 2001; 10(2): 101-104.

[9] Huang YS. Research progress on the prevention of organ damage in the early stage of burns. Chinese Journal of Burns. 2003; 19(5): 257-260. 